\title{
Impact of System Factors on the Performance of Photoacoustic Tomography Scanners
}

\author{
Chao Tian $\odot,{ }^{1,2,{ }^{*}}$ Mengliu Pei, ${ }^{1,2}$ Kang Shen, ${ }^{1,2}$ Songde Liu, ${ }^{1,2}$ Zhiming Hu, ${ }^{1,2}$ and Ting Feng ${ }^{3, \dagger}$ \\ ${ }^{1}$ Department of Precision Machinery and Precision Instrumentation, University of Science and Technology of \\ China, Hefei, Anhui 230026, China \\ ${ }^{2}$ Key Laboratory of Precision Scientific Instrumentation of Anhui Higher Education Institutes, University of \\ Science and Technology of China, Hefei, Anhui 230026, China \\ ${ }^{3}$ School of Electronic and Optical Engineering, Nanjing University of Science and Technology, \\ Nanjing, Jiangsu 210094, China
}

(Received 30 May 2019; revised manuscript received 25 August 2019; published 2 January 2020)

\begin{abstract}
High-performance imaging is essential for widespread applications of photoacoustic tomography (PAT) in biomedicine. So far, no comprehensive studies are reported on the impact of system factors on imaging performance, in spite of their importance. Based on a prototype PAT scanner, we study eight factors associated with the acoustic reception process in PAT, namely, detector view angle, element number, center frequency, bandwidth, aperture size, focusing, orientation error, and scan step angle error, and investigated how they impact on the image quality. Simulations and experiments are both presented to support the findings in this study. This work is expected to provide a practical guide for advanced PAT scanner design with enhanced imaging performance.
\end{abstract}

DOI: 10.1103/PhysRevApplied.13.014001

\section{INTRODUCTION}

Based on the energy conversion of light into sound, photoacoustic tomography (PAT) is an emerging noninvasive biomedical imaging technique that has experienced explosive developments in the past two decades [1]. As a hybrid imaging technique, PAT possesses molecular contrast due to optical absorption found in optical imaging, while maintaining high spatial resolution in deep tissue found in ultrasound imaging $[2,3]$. PAT can visualize biological samples at scales from organelles, cells, tissues, and organs to the small-animal whole body and can reveal multidimensional biological information, such as anatomical, functional, molecular, genetic, and metabolic data [1]. PAT has unique applications in a range of biomedical fields [4,5], such as cell biology [6-9], neurology [10-12], oncology [13,14], rheumatology [15], and ophthalmology $[16,17]$. Both basic sciences and clinical translations involving PAT are expected to grow in the future.

\footnotetext{
*ctian@ustc.edu.cn

${ }^{\dagger}$ fengting@njust.edu.cn
}

Published by the American Physical Society under the terms of the Creative Commons Attribution 4.0 International license. Further distribution of this work must maintain attribution to the author(s) and the published article's title, journal citation, and DOI.
In PAT, nanosecond laser pulses are used to illuminate biological tissues and excite ultrasound emissions from optical absorbing chromophores. Ultrasound detectors are then employed to receive the ultrasound signal to form images through acoustic inversion. Since the light-tosound energy conversion efficiency is limited and the illumination laser fluence should be controlled within the safety standard [18], the resulting photoacoustic pressure is typically weak, on the order of tens of thousands of pascals [19]. To achieve high-performance imaging in practical biomedical applications, it is essential to properly design the PAT systems for optimal signal reception and image quality.

Several groups have performed pioneering studies in exploring critical system parameters, such as detector bandwidth and aperture size, on final image quality in PAT. Xu and Wang first proposed analytical relationships between image spatial resolution and ultrasound detector bandwidth, as well as aperture size, using the point spread function (PSF) theory [20]. Wang et al. further interpreted the relations in the Fourier domain and drew similar conclusions [21]. Xu et al. theoretically studied how limited detection angle would affect final image quality and defined the concept of the "detection region" for the optimal design of the geometry of the detector [22]. Later, Haltmeier and Zangerl derived analytical expressions of the PSF to deal with limited bandwidth and finite aperture size of approximate point and line detectors and showed 
that the PSF, due to limited bandwidth, was spatially invariant for both detectors, while the PSF due to finite aperture size was only spatially invariant for line detectors [23]. Most recently, Dima et al. demonstrated the imaging performance of PAT systems employing 64, 128, and 256 ultrasound detectors and concluded that images acquired using 256 detectors had significantly higher quality than those acquired using 128 or 64 detectors [24]. These studies lay the foundations of image-quality enhancement in PAT. However, most of the studies focus on theoretical aspects or only cover specific topics.

Here, we study eight system factors associated with a typical PAT scanner and demonstrate how they affect the image quality from numerical and experimental perspectives. The eight factors, namely, detector view angle, element number, center frequency, bandwidth, aperture size, focusing, orientation error, and scan step angle error, all relate to the acoustic reception process and influence the final image quality, to some extent. The study is carried out based on a prototype PAT scanner, where a single-element detector is rotated around the sample to capture generated photoacoustic signals. The method, results, discussion, and conclusions are presented below.

\section{METHODS}

The prototype PAT scanner (Fig. 1) works as follows. Nanosecond-duration near-infrared light pulses emitted from the tunable laser system are used to illuminate the sample for photoacoustic signal excitation. The excited signals propagate in free space and are picked up by an ultrasound detector, which is driven by a step motor and rotates around the sample. The acquired photoacoustic projections are then digitized by an oscilloscope and transferred to a computer for image reconstruction. The sample and ultrasound detector are both immersed in water for optimal ultrasound coupling. This single-detector-based prototype PAT scanner was demonstrated to be a success in early photoacoustic studies $[25,26]$. Even today, it is still frequently used in many photoacoustic experiments for proof-of-concept studies due to its simplicity, low cost, and effectiveness [27]. Although imaging speed and resolution are greatly enhanced, modern PAT scanners work similarly to this prototype $[24,28]$. System parameters study based on this prototype PAT scanner is thus of general significance.

In the prototype system, if the sample is uniformly illuminated, the imaging performance will be mainly determined by the acoustic reception process. Eight system parameters associated with the ultrasound detector, namely, view angle, element number, center frequency, bandwidth, aperture size, focusing, orientation error, and scan step angle error, are studied to understand how they independently influence the final imaging performance. Among the eight parameters, the first two relate to the arrangement of the detector, the middle four define the properties of the detector, while the last two describe the alignment accuracy of the detector. The method used in this study is based on single-variable analysis, which means that, in each analysis, only one parameter is allowed to vary and all others are kept in ideal situations, unless otherwise stated. The single-variable analysis method allows us to individually study the effect of each variable on final imaging performance. Moreover, in all numerical studies presented here, forward and inverse acoustic processes, including signal generation, propagation, and reception, are simulated using the $k$-wave toolbox [29]. In all experimental studies, the inverse image reconstruction process is performed based on the filtered back-projection algorithm [30].

To better illustrate findings in the study, we design four different phantoms used in numerical and experimental analyses: a blood vessel pattern, a multidisk pattern, a dot grid pattern, and a human hair cross, as shown in Fig. 2. The blood vessel pattern (pattern width, approximately $30 \mathrm{~mm}$; trunk width, approximately $0.8 \mathrm{~mm}$; tip width,

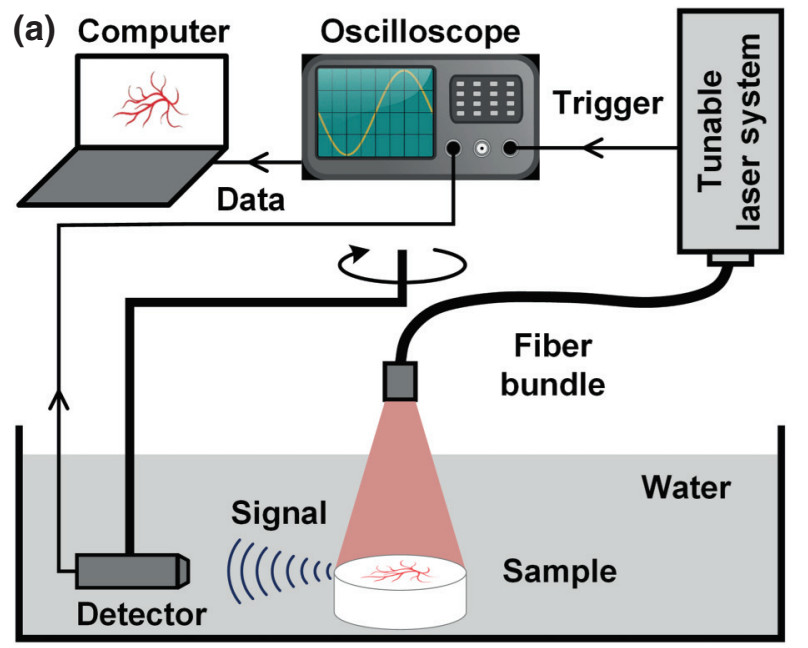

(b)

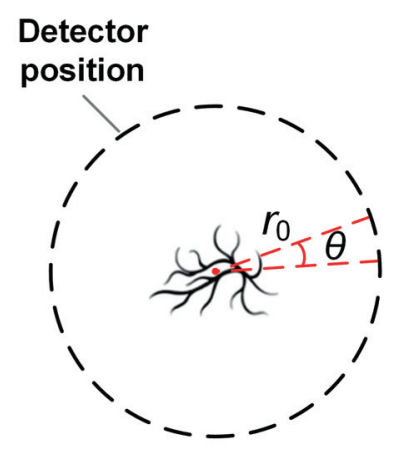

FIG. 1. The prototype PAT scanner. (a) Schematic. (b) Scanning trajectory of the single-element detector. $r_{0}$ denotes detection radius; $\theta$ denotes scan step angle. 


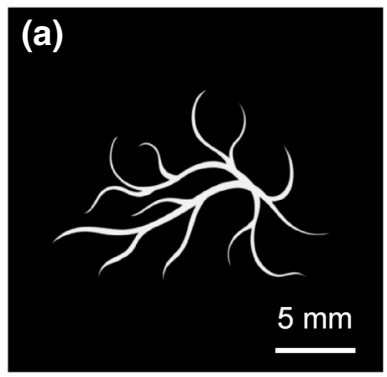

(b)

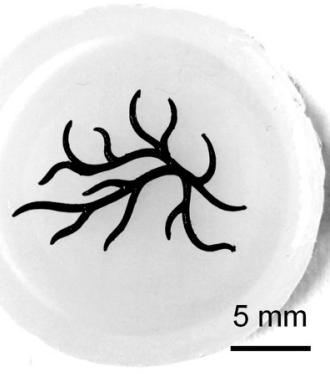

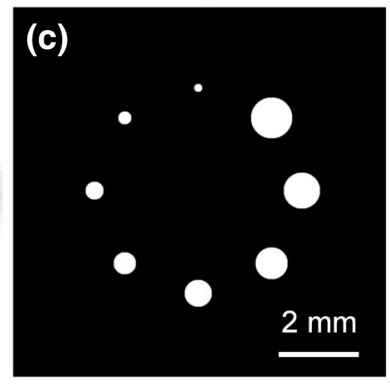

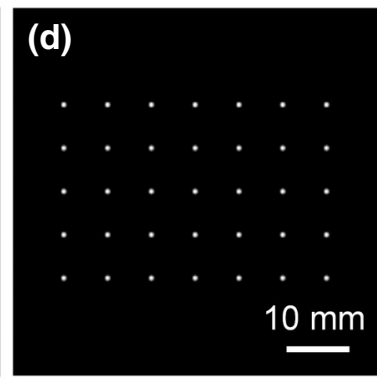

FIG. 2. Numerical and experimental phantoms used in this study. (a) Numerical blood vessel phantom (total width, approximately $30 \mathrm{~mm}$; vessel trunk width, approximately $0.8 \mathrm{~mm}$; tip width, approximately $0.3 \mathrm{~mm}$ ). (b) 3D-printed experimental blood vessel phantom. (c) Numerical multidisk phantom with uniformly-varying diameters (minimum diameter, $0.2 \mathrm{~mm}$; maximum diameter, $1 \mathrm{~mm}$ ). (d) Numerical dot grid phantom with five rows and seven columns (dot diameter, $1.2 \mathrm{~mm}$ ).

approximately $0.3 \mathrm{~mm}$ ) is mainly intended to evaluate the performance of the imaging system in a general sense and has both numerical and physical versions [Figs. 2(a) and 2(b)]. The physical version is 3D-printed based on the numerical model, but is slightly thicker, especially around vessels tips, due to limited printing resolution. The disk pattern [Fig. 2(c)] has multiple disks (minimum diameter, $0.2 \mathrm{~mm}$; maximum diameter, $1 \mathrm{~mm}$ ) with uniformly varying diameters and is mainly used to evaluate the effects of detector bandwidth and center frequency on imaging results. The dot grid pattern [Fig. 2(d)] has uniformly distributed dots (diameter, $1.2 \mathrm{~mm}$ ) aligned in five rows and seven columns and is used to investigate the effects of detector aperture size and detector focusing characteristics. The hair cross phantom, not shown here, is made up of two human hairs (diameter, approximately $80 \mu \mathrm{m}$ ) and is used to experimentally evaluate spatial resolutions of the prototype PAT scanner. The four phantoms in combination can sufficiently evaluate the imaging performance of the PAT scanner in various situations. In addition, four ultrasound detectors with different center frequencies and different focusing characteristics are employed in experiments with specifications detailed in Table I.

\section{RESULTS AND DISCUSSION}

\section{A. Detector view angle $(\Omega)$}

The detector view angle, $\Omega$, refers to the angle enclosed by the detector during the measurement and has a profound impact on final image quality. Ideally, the detector should completely enclose the sample for perfect image reconstruction; thus, indicating a $4 \pi$ steradian view angle $(\Omega=4 \pi)$ for three-dimensional (3D) detection and a $2 \pi$ radian view angle $(\Omega=2 \pi)$ for two-dimensional (2D) detection are preferred. However, in real scenarios, such as breast imaging and skin imaging, the view angle is often limited and images need to be recovered from incomplete projections. The image reconstruction problem in limitedview PAT can be well illustrated by means of the detection region concept developed by $\mathrm{Xu}$ et al. [22], which is defined as the convex hull of the detector arc. As such, a photoacoustic source can be divided into two reconstruction parts: one inside the detection region and the other outside the detection region. The one inside the detection region is the area that any line passing through it intersects with the detection surface, as shown in Fig. 3. Otherwise, it is regarded as outside of the detection region. For photoacoustic structures inside the detection region or outside the detection region but with their normal intersecting with the detection surface, they can be stably recovered. Otherwise, details of the photoacoustic source will be lost.

Figure 3 illustrates a numerical and experimental example demonstrating limited- and full-view PAT imaging. The blood vessel phantom is imaged using a point ultrasound detector, which rotates around the phantom for $90^{\circ}$, $180^{\circ}, 270^{\circ}$, and $360^{\circ}$. It is easy to see that a larger view angle results in better reconstruction results. The $360^{\circ}$ full-view detection scheme gives the highest reconstruction quality, as expected. In the $270^{\circ}$ case, although the

TABLE I. Specifications of the four ultrasound detectors used in the experiments.

\begin{tabular}{llcrr}
\hline \hline $\begin{array}{l}\text { Center frequency } \\
(\mathrm{MHz})\end{array}$ & Focusing & $\begin{array}{c}-6 \mathrm{~dB} \text { two-way } \\
\text { bandwidth (\%) }\end{array}$ & $\begin{array}{c}\text { Aperture size } \\
{[\mathrm{mm} \text { (inch)] }}\end{array}$ & $\begin{array}{l}\text { Model No. } \\
1\end{array}$ \\
\hline 1 & Flat & 56 & $12.7(0.5)$ & V303, Olympus \\
2.25 & Flat & 64 & $9.5(0.375)$ & V325, Olympus \\
5 & Flat & 63 & $9.5(0.375)$ & V326, Olympus \\
5 & Focused (focal length $50.8 \mathrm{~mm})$ & 69 & $12.7(0.5)$ & V309, Olympus \\
\hline \hline
\end{tabular}



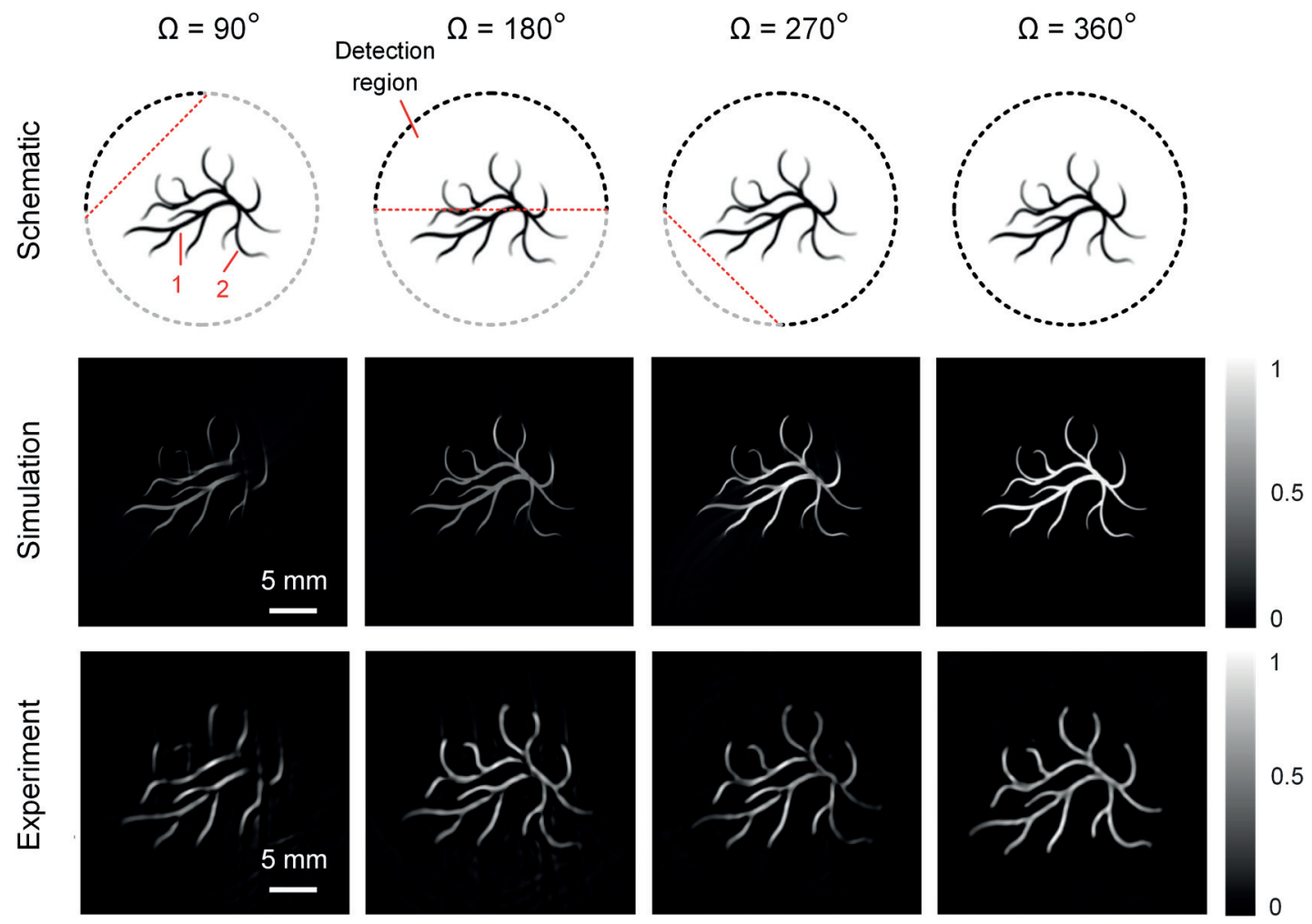

FIG. 3. The effect of detector view angle on image quality. First row: schematic showing relative positions of the detector and blood vessel sample at different view angles. Second row: simulation results showing images reconstructed at different measurement view angles (point detector, full bandwidth, $r_{0}=30 \mathrm{~mm}$ ). Third row: experimental results showing images reconstructed at different measurement view angles using an ultrasound detector (Model: V303, center frequency $f_{c}=1 \mathrm{MHz}, r_{0}=100 \mathrm{~mm}$ ). The result in the $270^{\circ}$ detector view angle is visually similar to that of $180^{\circ}$, but has significantly fewer background artifacts. First to fourth columns, measurement view angles of $\Omega=90^{\circ}, 180^{\circ}, 270^{\circ}$, and $360^{\circ}$, respectively.

blood vessels are not completely enclosed by the detection surface, they can still be stably reconstructed, since they are within the detection region defined above. This finding may inspire innovative designs of ultrasound detection schemes. Some modern PAT scanners adopt the $270^{\circ}$ detection strategy and can reduce the detector fabrication cost, while producing outstanding image quality [24]. In the $90^{\circ}$ case, the blood vessels are totally outside the detection region. The vessels (e.g., vessel 1) parallel with the detection surface can be reasonably recovered, while those (e.g., vessel 2) perpendicular to the detection surface are lost after reconstruction; this is in agreement with the aforementioned rules. The numerical simulations in the second row of Fig. 3 are well supported by the experimental results in the third row.

\section{B. Detector number $(n)$}

Ideally, the number of detectors, $n$, should be as large as possible, to receive generated ultrasound signals from the sample for accurate image reconstruction. This will not only improve spatial resolution, but also enhance imaging sensitivity. However, in reality, it is impossible for a PAT scanner to employ an infinite number of detectors due to high cost, fabrication complexity, and limited detection space. Determining an optimal number of detectors is of great importance to achieve high-performance imaging at an affordable cost. Dima et al. observed the problem and studied the effect of transducer number [24].

Figure 4 illustrates a study showing the impact of the detector number, $n$, on the image quality. The first row is a group of simulations that demonstrate how the number of detectors correlates with the imaging performance. It is seen that with an increase of the detector number from $n=32$ to $n=512$, reconstruction artifacts produced at sparse detectors are well suppressed and the final image quality improves significantly. The second row shows the corresponding experimental results under similar measurement conditions, except with a $1 \mathrm{MHz}$ detector (V303). The experimental results are consistent with the numerical simulations for most detector numbers, except for $n=256$ and $n=512$, where the image reconstructed at 512 detectors seems to have little improvement compared with that at 256 detectors. The justification is that, in real experiments, the physical size of the detector may make the 


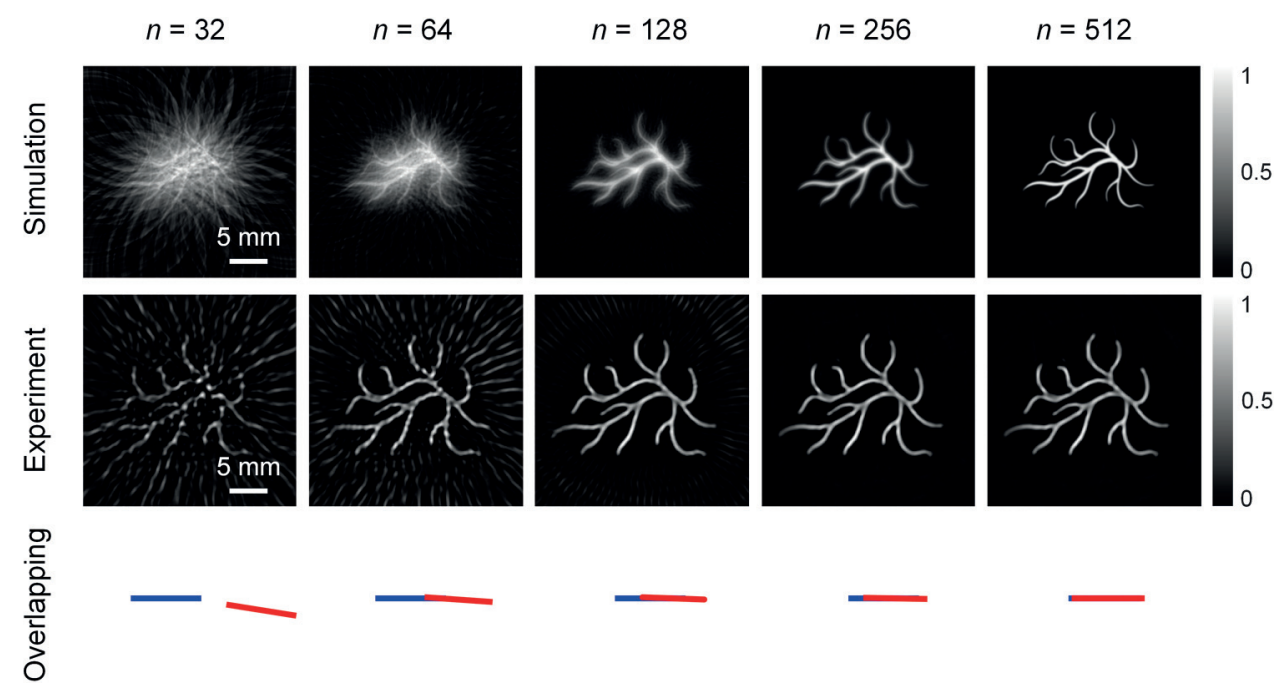

FIG. 4. The effect of the detector number on image quality. First row: simulation results showing the blood vessel phantom reconstructed using different detector numbers (point detector, full bandwidth, $r_{0}=30 \mathrm{~mm}$ ). Second row: experimental results showing the blood vessel phantom reconstructed using different detector numbers (Model: V303; aperture size, $12.7 \mathrm{~mm}$; center frequency, $f_{c}=1 \mathrm{MHz} ; r_{0}=100 \mathrm{~mm}$ ). Third row: aperture overlapping of the detector at two consecutive scan positions. First to fifth columns, detector numbers of $n=32,64,128,256$, and 512, respectively.

signal reception surfaces at two consecutive scan positions overlap, resulting in significantly redundant information when the detector number is large. In this case, the aperture size of the detector is $12.7 \mathrm{~mm}$ and the overlap ratio is $90.3 \%$ for $n=512$, as shown in the third row of Fig. 4 . In practical applications, 256 detectors evenly distributed in a full circle is a good choice for the sensor number to balance the imaging performance and cost.

\section{Detector center frequency $\left(f_{c}\right)$}

The detector view angle, $\Omega$, and the detector number, $n$, discussed above both relate to the arrangement of a chosen detector and are critical to the imaging performance of a PAT scanner. In addition, the properties of the chosen detector, including the center frequency, $f_{c}$; the bandwidth, $B$; the aperture size, $2 a$; and the focusing characteristics, $l_{n}$, are also of great importance. To achieve high-performance imaging, the center frequency of the detector, $f_{c}$, should be selected based on two facts. First, the center frequency of the detector should match the desired spatial resolution [31]. Detectors with higher center frequencies generally have better spatial resolving ability, and thus, can produce images with greater detail, as demonstrated by $\mathrm{Ku}$ et al. [32]. Second, the center frequency should match the frequency contents of generated photoacoustic signals to achieve maximum signal reception sensitivity. Although photoacoustic signals are broadband, they have limited bandwidths in the Fourier domain and typically center at a certain frequency (called center frequency, $f_{c}$ ). Take a spherical absorber with a diameter of $d$ as an example, the center frequency, $f_{c}$, of its generated photoacoustic signals can be estimated as

$$
f_{c}=\frac{v_{s}}{1.5 d}
$$

where $v_{s}$ is the speed of sound in the medium. A smaller absorber will result in a higher center frequency. Figure 5 illustrates this idea based on three uniform disks with diameters of $1 \mathrm{~mm}, 200 \mu \mathrm{m}$, and $50 \mu \mathrm{m}$. Figures $5(\mathrm{a})$ and 5(b) show corresponding time-domain and frequencydomain photoacoustic signals, from which the center frequencies are estimated as 1,5 , and $20 \mathrm{MHz}$ for the $1 \mathrm{~mm}$, $200 \mu \mathrm{m}$, and $50 \mu \mathrm{m}$ diameter disks, respectively, assuming the speed of sound in the medium of $v_{s}=1500 \mathrm{~m} / \mathrm{s}$.

The first row of Fig. 6 is a group of simulations showing the blood vessel phantom imaged using ultrasound detectors $(100 \%$ bandwidth) with center frequencies of $1,2.25$, and $5 \mathrm{MHz}$, respectively. The $1 \mathrm{MHz}$ detector produces the brightest image, but the worst resolution, while the $5 \mathrm{MHz}$ detector gives the best resolution, but the darkest image due to loss of low-frequency components. This is easy to understand because the center frequency of the photoacoustic signal of the blood vessels (single vessel width, $0.8 \mathrm{~mm}$ ) is estimated to be around $1.25 \mathrm{MHz}$, which is closer to the frequency response of the $1 \mathrm{MHz}$ detector in the simulation. Experimental results shown in the second row of Fig. 6 support this finding.

To further illustrate this point, we perform another set of simulations and experiments using the multidisk phantom and the human hair cross. The first row of Fig. 7 is the simulation showing that the multidisk phantom is imaged using detectors ( $200 \%$ bandwidth) with center frequencies of 1.5, 2.5, 5, and $10 \mathrm{MHz}$. Results reveal that a higher 

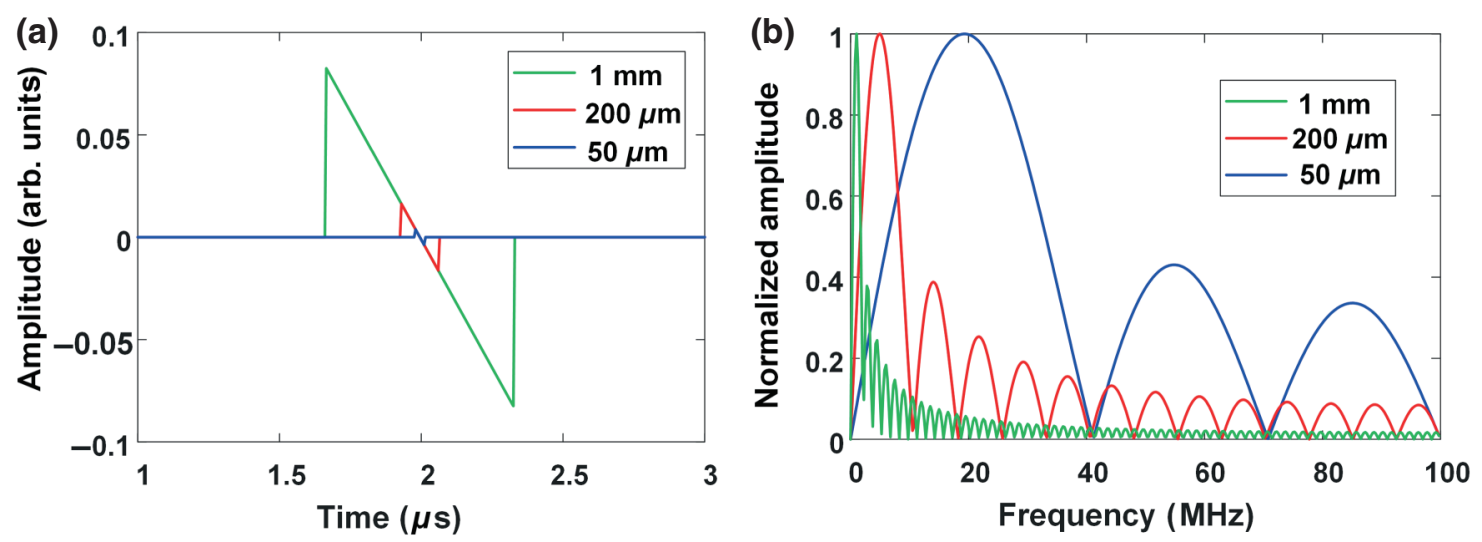

FIG. 5. Photoacoustic signals of uniform disks and their spectra. (a) Time-domain $\mathrm{N}$-shaped photoacoustic signals of three disks with diameters of $1 \mathrm{~mm}, 200 \mu \mathrm{m}$, and $50 \mu \mathrm{m}$. (b) Corresponding Fourier spectra.

center frequency will result in a sharper image. The second row of Fig. 7 is the experiment designed to quantitatively calibrate the spatial resolution of the PAT scanner, when equipped with detectors with center frequencies of $1 \mathrm{MHz}$ (V303), $2.25 \mathrm{MHz}$ (V325), and $5 \mathrm{MHz}$ (V326) based on the human hair cross (hair diameter, approximately $80 \mu \mathrm{m}$ ). By subtracting the hair diameter from the widths of the main lobes of the signals [32], the spatial resolutions of the PAT system using the three detectors are estimated to be $410 \mu \mathrm{m}(1 \mathrm{MHz}), 260 \mu \mathrm{m}(2.25 \mathrm{MHz})$, and $140 \mu \mathrm{m}(5 \mathrm{MHz})$. The results again illustrate that ultrasound detectors with higher center frequencies have better spatial resolving power.

\section{Detector bandwidth $(B)$}

As mentioned above, photoacoustic signals are typically broadband. However, an ultrasound detector used to receive photoacoustic signals usually has limited frequency response, as characterized by the lower cutoff frequency, $f_{1}$; the upper cutoff frequency, $f_{2}$; and the center frequency, $f_{c}$, as shown in Fig. 8(a). The limited bandwidth characteristics of an ultrasound detector directly impact on the PSF and the spatial resolution of the detection system. According to the theoretical analysis by Xu and Wang [20], if the frequency response of an ultrasound detector is flat between $f_{1}$ and $f_{2}$, the PSF can be formulated as

$$
h(r)=\frac{k_{2}^{3}}{2 \pi^{2}} \frac{j_{1}\left(k_{2} r\right)}{k_{2} r}-\frac{k_{1}^{3}}{2 \pi^{2}} \frac{j_{1}\left(k_{1} r\right)}{k_{1} r},
$$

where $k_{1}=2 \pi f_{1} / v_{s}$ and $k_{2}=2 \pi f_{2} / v_{s} ; v_{s}$ is the speed of sound, and $j_{1}$ is the first-order spherical Bessel function of the first kind. Assuming that the frequency response of the detector is symmetric and using the relationships $k_{1}=(1-0.5 B) k_{c}$ and $k_{2}=(1+0.5 B) k_{c}$, the PSF can be represented by the center frequency, $f_{c}$, and the bandwidth, $B$, as

$$
\begin{aligned}
h(r)= & \frac{k_{c}^{2}}{2 \pi^{2} r}\left\{(1+0.5 B)^{2} j_{1}\left[(1+0.5 B) k_{c} r\right]\right. \\
& \left.-(1-0.5 B)^{2} j_{1}\left[(1-0.5 B) k_{c} r\right]\right\}
\end{aligned}
$$

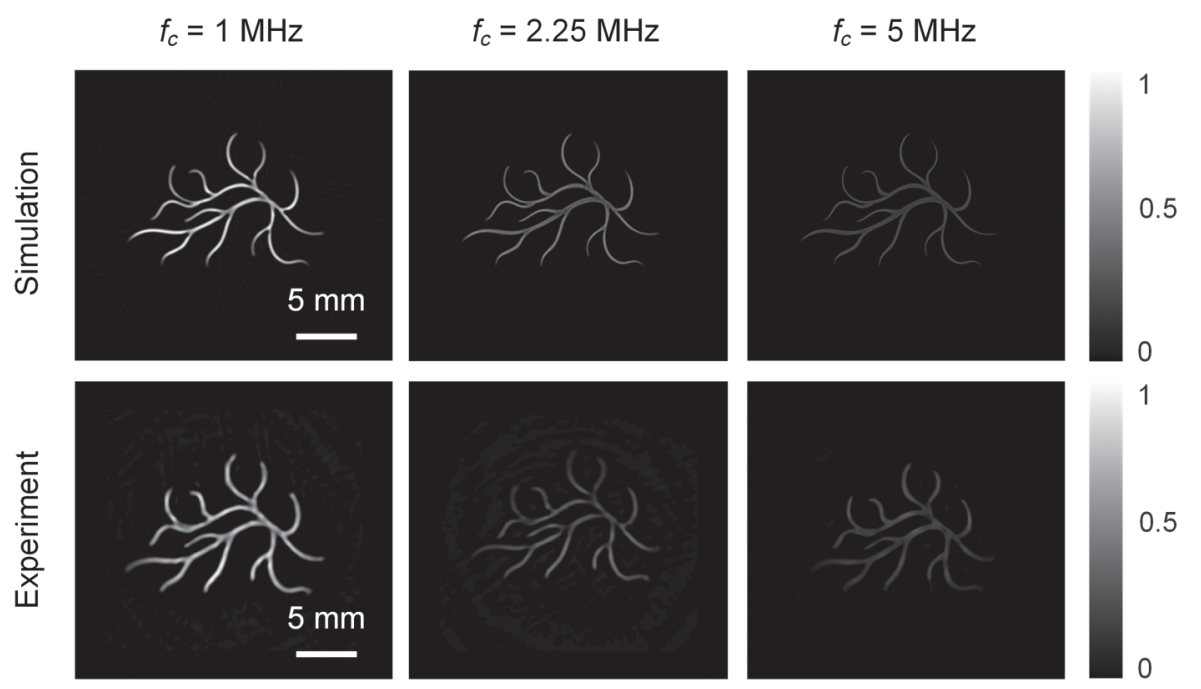

FIG. 6. The effect of detector center frequency on image quality. First row: simulation results showing the blood vessel phantom reconstructed using detectors with different center frequencies (point detector, bandwidth $\left.B=100 \%, r_{0}=30 \mathrm{~mm}\right)$. Second row: experimental results showing the blood vessel phantom reconstructed using detectors with different center frequencies (Model: V303, V325, V326, $r_{0}=100 \mathrm{~mm}$ ). First to third columns, detector center frequencies of $f_{c}=1$, 2.25 , and $5 \mathrm{MHz}$, respectively. 

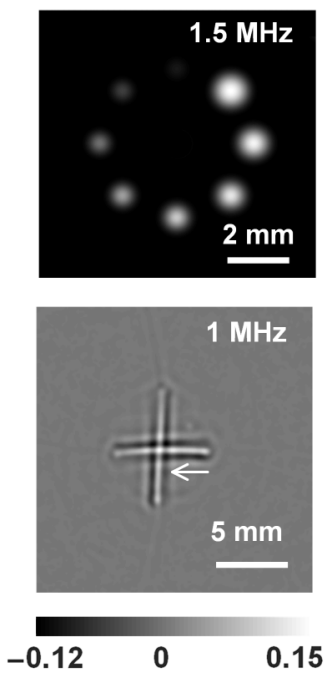
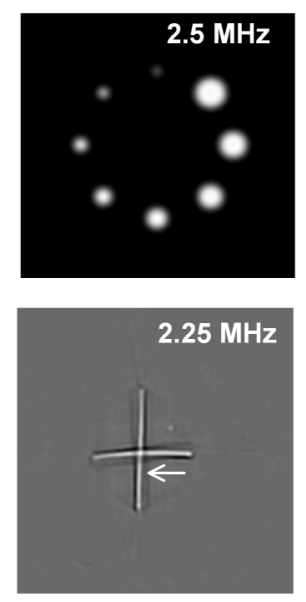

$0.15-0.15$
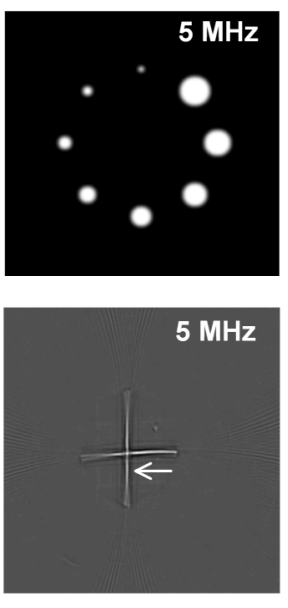

$0.23-0.130$
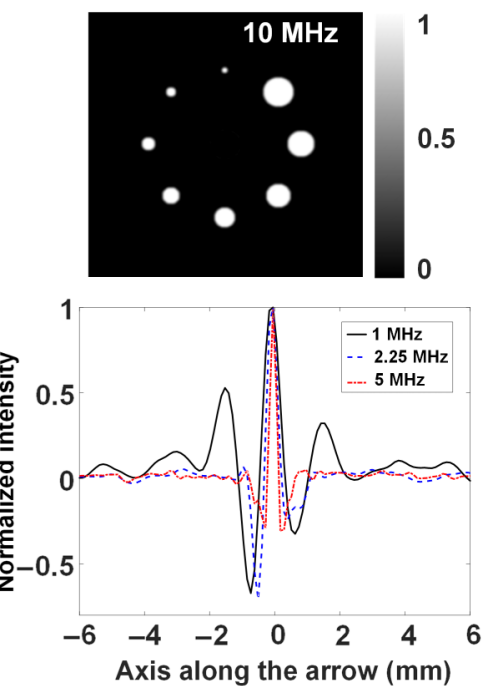

FIG. 7. The effect of the detector center frequency on lateral resolution. First row: simulation results showing the multidisk phantom imaged using detectors with center frequencies of $1.5,2.5,5$, and $10 \mathrm{MHz}$ (point detector, bandwidth $B=200 \%$ ). Second row: experimental results showing the human hair cross phantom imaged using detectors with center frequencies of $1 \mathrm{MHz}$ (V303), 2.25 MHz (V325), and $5 \mathrm{MHz}$ (V326), and a comparison of intensity profiles of the rows indicated by the arrows. Detectors with higher center frequency produce sharper images.

where $k_{c}=2 \pi f_{c} / v_{s}$. Since the spatial resolution is usually represented by the full width at half maximum (FWHM) of the PSF, Eq. (3) indicates that the spatial resolution of a PAT system is determined by the center frequency, $f_{c}$, and the bandwidth, $B$, of the detector. For typical detectors with $80 \%$ bandwidth $\left[\left(f_{2}-f_{1}\right) / f_{c}=80 \%\right]$, the spatial resolution limit is estimated to be $0.55 \lambda_{c}$, where $\lambda_{c}$ is the center wavelength. Figure 8(b) plots the PSFs of three ultrasound detectors with bandwidths of $50 \%, 100 \%$, and $150 \%\left(f_{c}=3 \mathrm{MHz}\right)$. It is easy to see that an ultrasound detector with a broader bandwidth has a narrower PSF, and thus, finer spatial resolution, while an ultrasound detector with a narrower bandwidth has a more oscillating PSF and more significant ring artifacts.

Figure 9 presents two groups of simulations, showing how ultrasound detector bandwidth would impact on the final image quality. The first row shows the imaging results of the blood vessel phantom using ultrasound detectors (center frequency, $f_{c}=1 \mathrm{MHz}$ ) with bandwidths of $50 \%$, $100 \%$, and $150 \%$, and full bandwidth. When the bandwidth of the ultrasound detector is set to $50 \%$, significant ring artifacts occur, which can be largely mitigated by increasing the bandwidth. The second row shows the imaging results of the multidisk phantom using ultrasound detectors with the same bandwidth setting. In addition to the conclusion that increasing bandwidth can mitigate ring artifacts, as described, we note two other interesting findings. First, ultrasound detectors with broader bandwidth provide better spatial resolution and sharper edges. Second, ultrasound detectors with broader bandwidth help to recover low-frequency components (the center parts of the disks) of the objects. Ultrasound detectors with broader bandwidths are favorable for PAT, which is consistent with previous results reported in Refs. [33,34]. In this example, (a)

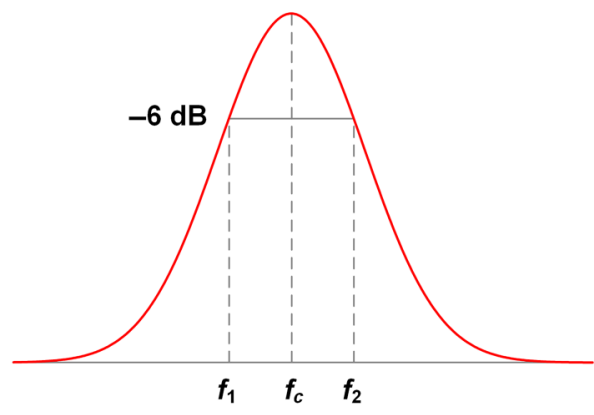

(b)

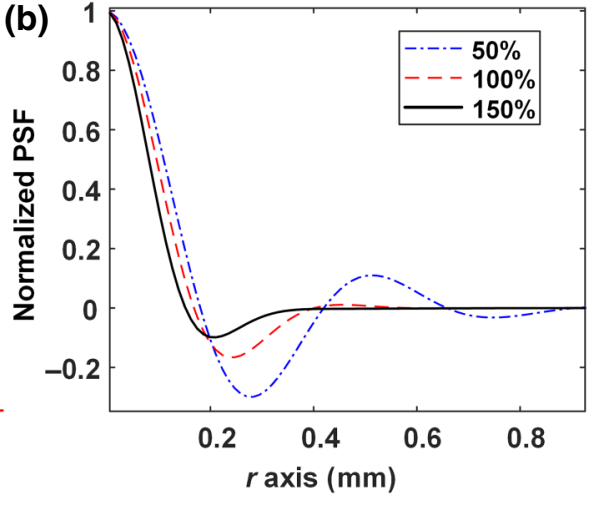

FIG. 8. (a) Bandpass characteristics of typical ultrasound detectors. (b) PSFs of ultrasound detectors with bandwidths of $50 \%, 100 \%$, and $150 \%\left(f_{c}=3 \mathrm{MHz}\right) . f_{1}$, lower cutoff frequency; $f_{2}$, upper cutoff frequency; $f_{c}$, center frequency. 

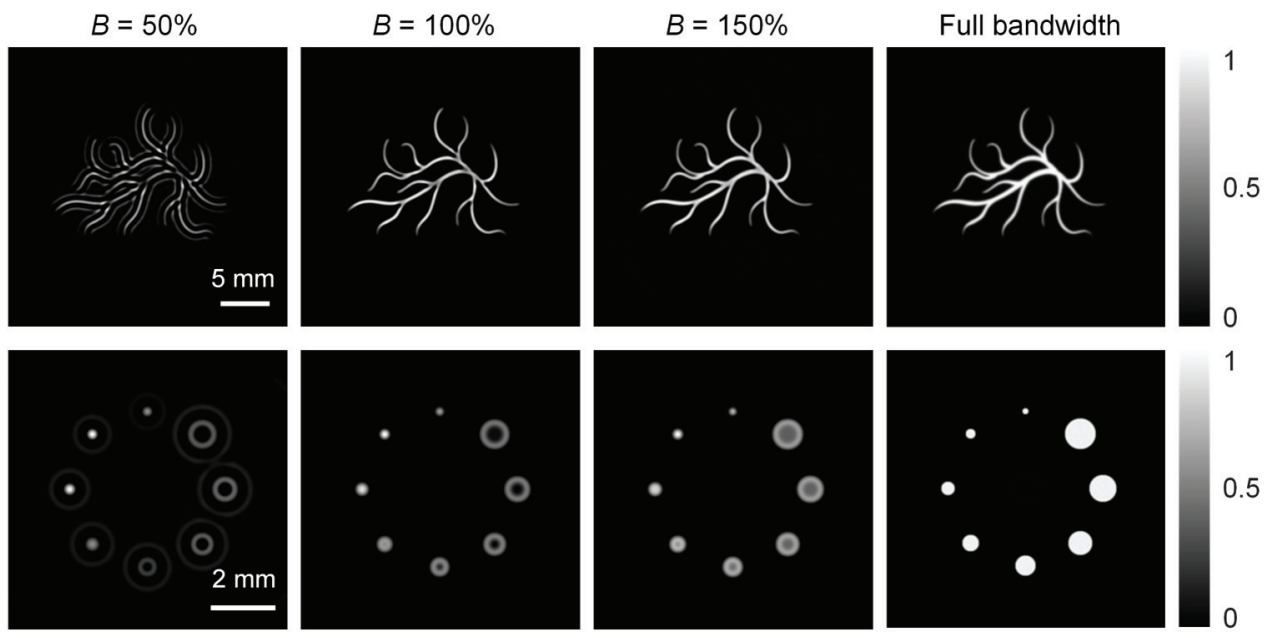

FIG. 9. The effect of detector bandwidth on image quality. First row: imaging results of the blood vessel phantom using detectors with different bandwidths $\left(f_{c}=1 \mathrm{MHz}, r_{0}=30 \mathrm{~mm}\right)$. Second row: imaging results of the multidisk phantom using detectors with different bandwidths $\left(f_{c}=3 \mathrm{MHz}, r_{0}=4.5 \mathrm{~mm}\right)$. First to fourth columns, detector bandwidths of $B=50 \%, 100 \%$, $150 \%$, and full, respectively. Detectors with broader bandwidths not only suppress ringing oscillation artifacts, but also produce sharper images.

only simulation is performed because physical detectors with different bandwidths (i.e., $50 \%, 100 \%$, and $150 \%$ in this case) are not readily available.

\section{E. Detector aperture size (2a)}

In PAT, detectors used for ultrasound signal reception usually have a finite aperture size, rather than being a point sensor. The finite aperture size $2 a$ impacts on the imaging performance of a PAT scanner mainly in two ways. First, it degrades the accuracy of the mathematical model used for image reconstruction. In the forward process [Fig. 10(a)], ultrasound signals are actually detected by the entire surface of the detector. In the inverse process [Fig. 10(b)], ultrasound detectors with a finite aperture size are typically regarded as a point detector to simplify the mathematical model used in most state-of-the-art image reconstruction algorithms, such as filtered back projection [30] and time reversal [29], which inevitably blurs reconstructed images. To ease this problem, Yang et al. proposed a ring-based ultrasonic virtual point detector to avoid potential image blurring [35]. Second, different from point detectors with an omnidirectional response, detectors with finite aperture size have directional responses; this means that they have different degrees of response to ultrasound signals coming from different directions. The response differences can be characterized by the directivity function, $D(\theta)$, which directly correlates with the aperture size of the detector $2 a$ and can be formulated as

$$
D(\theta)=\frac{2 J_{1}(k a \sin \theta)}{k a \sin \theta},
$$

where $k=2 \pi / \lambda$ is the wave number, $\lambda$ is the wavelength, $\theta$ is the angle, and $J_{1}$ is the first-order Bessel function of the first kind for cylindrical coordinates. Figure 11 illustrates an example showing the directivity patterns of four ultrasound detectors with different aperture sizes, when $2 a / \lambda=1,2,4$, and 6.2. For a fixed ultrasound wavelength, $\lambda$, a larger aperture size $2 a$ will result in a more directional detector. The final impacts of the detector aperture size on the lateral resolution of the PAT scanner in Fig. 1 are (a)

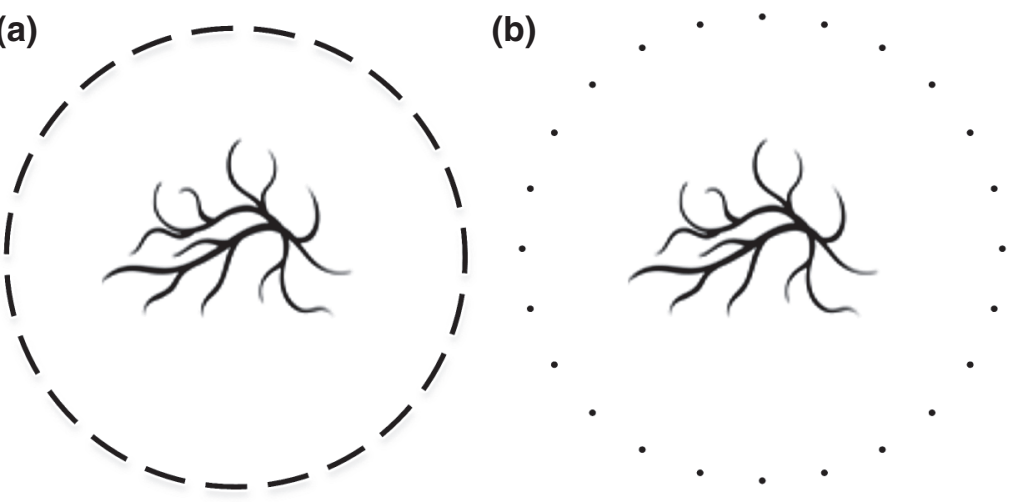

FIG. 10. Forward signal detection and inverse image reconstruction in PAT. (a) In the forward process, the received signal is the spatial integration of the pressure wave over the whole detector surface. (b) In the inverse process, the detector is simplified as a point to facilitate the subsequent image reconstruction procedure. 
(a)

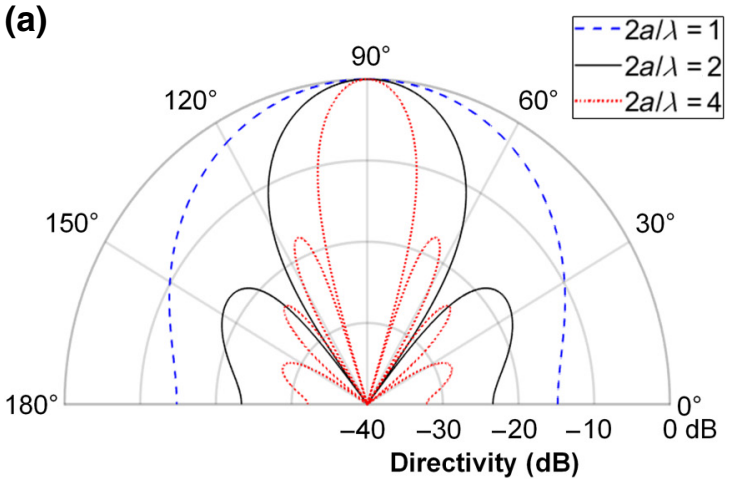

explicitly given by $\mathrm{Xu}$ and Wang [20] as

$$
\Delta l=2 a \frac{r}{r_{0}},
$$

where $2 a$ is the aperture size; $r_{0}$ is the detection radius; $r$ is the spatial coordinate of the image. The lateral resolution (LR) linearly degrades with the distance $r$ between the object and the center of the detection surface, indicating that, in the prototype PAT scanner, the detector aperture size will degrade the image quality at the edge more significantly than that in the center.

The first row of Fig. 12 is an experimental study demonstrating how the detector aperture size would impact on the image quality of the blood vessel phantom. The ultrasound detector used in the experiment is a $1 \mathrm{MHz}$ sensor with an aperture size of $2 a=12.7 \mathrm{~mm}$ (V303) and a detection radius of $r_{0}=25 \mathrm{~mm}$. The reconstructed image displayed in the first column in Fig. 12 shows that, while the central region of the blood vessels is well recovered, the edge part is blurred significantly due to the aperture effect of the detector. To confirm that blurring is indeed caused by the aperture, the aperture size of the detector is equivalently halved via increasing the detection radius, $r_{0}$, from
FIG. 11. Directivity patterns of ultrasound detectors with an aperture size $2 a$. (a) Directivity patterns in polar form, when $2 a / \lambda=1,2$, and 4. (b) Directivity pattern in $3 \mathrm{D}$, when $2 a / \lambda=6.4$.

25 to $50 \mathrm{~mm}$ and $100 \mathrm{~mm}$, for which the reconstructed images are shown in the second and third columns, respectively, of the first row of Fig. 12. Results reveal that, by decreasing the aperture size, the image quality can be greatly enhanced, especially at the edge. The second row of Fig. 12 is the corresponding numerical study under similar conditions $\left(f_{c}=1 \mathrm{MHz}, 2 a=12.7 \mathrm{~mm}, r_{0}=25,50\right.$, and $100 \mathrm{~mm}$ ), which is consistent with the experimental results.

To further illustrate the detector aperture effect, we perform another group of simulations, to compare images produced by pointlike detectors and nonpointlike detectors. Figure 13(a) shows the original dot grid pattern (dot diameter: $1.2 \mathrm{~mm}$ ) and Figs. 13(b) and 13(c) present the imaging results using a point detector and a detector with an aperture size of $2 a=3 \mathrm{~mm}$, respectively. The pointlike detector-based imaging almost reproduces the original pattern, while the nonpointlike detector-based imaging causes severe distortion and blurring at the edge of the image, especially in the tangential direction, as shown in Figs. 13(d) and 13(e). In this example, only simulation results are presented because point detectors, which are required for the experiments, are not readily available.
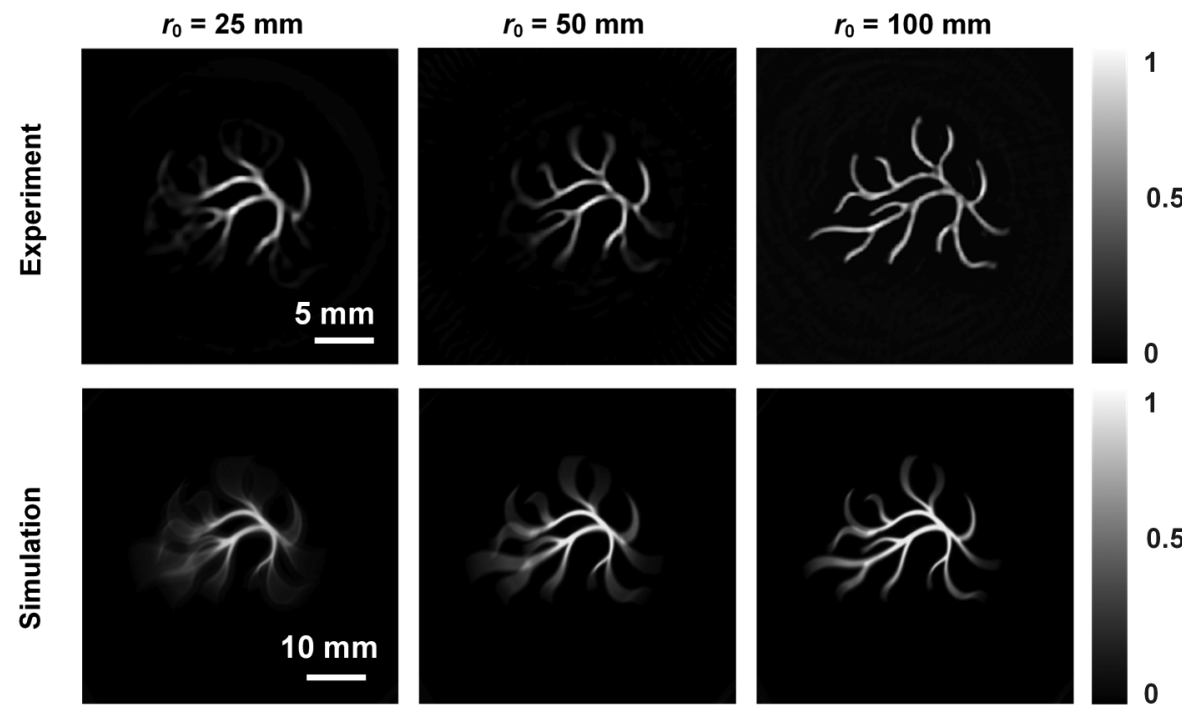

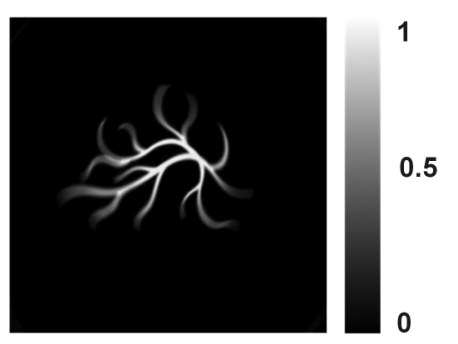

FIG. 12. The effect of detector aperture size on image quality. First row: experimental results showing the blood vessel phantom imaged using an ultrasound detector at different detection radii, $r_{0}$ (Model: V303, aperture size $2 a=12.7 \mathrm{~mm}, f_{c}=1 \mathrm{MHz}$ ). Second row: simulation results showing the blood vessel phantom imaged using an ultrasound detector at different detection radii, $r_{0}$ (aperture size $2 a=12.7 \mathrm{~mm}$, $f_{c}=1 \mathrm{MHz}, B=$ full bandwidth). First to third columns, detection radii of $r_{0}=25$, 50 , and $100 \mathrm{~mm}$, respectively. 

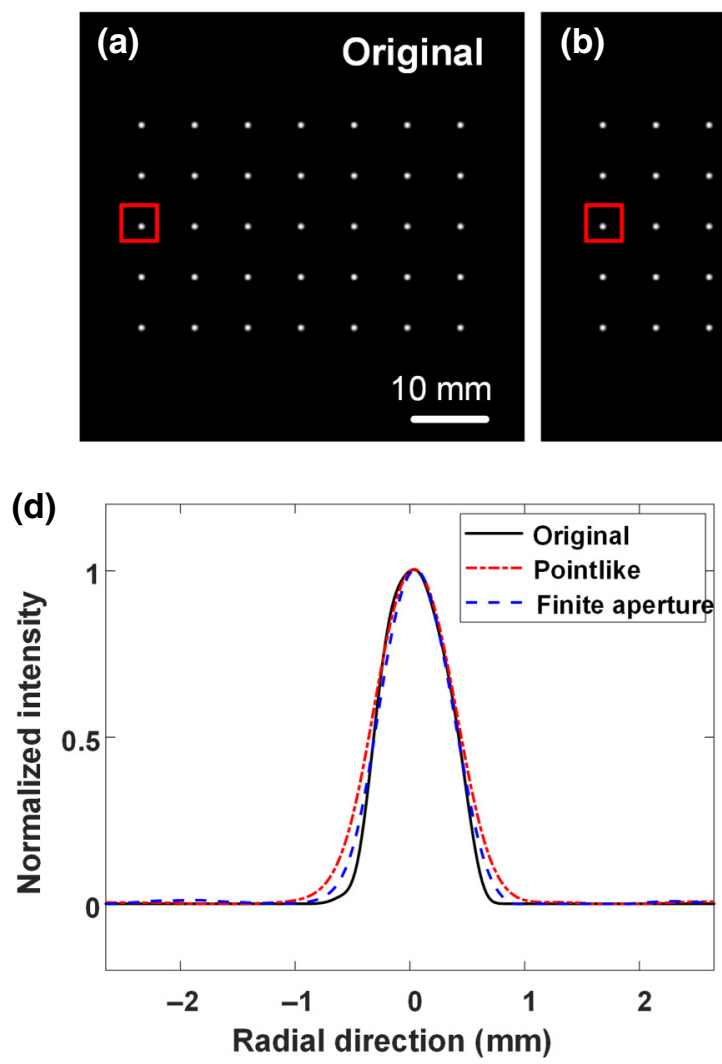
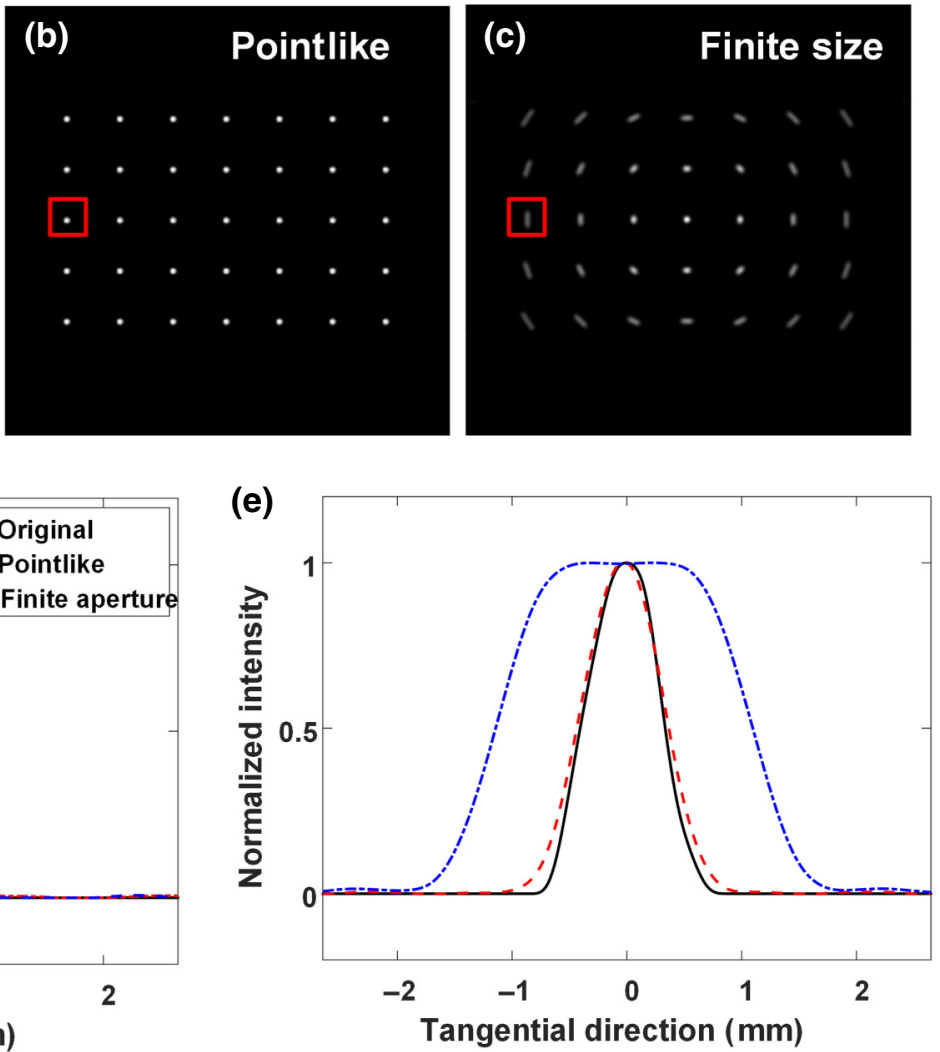

FIG. 13. The effect of detector aperture size on image resolution. (a) Dot grid phantom used in the simulation (dot diameter, $1.2 \mathrm{~mm}$ ). (b) Reconstructed image using a point detector $\left(n=512, B=\right.$ full bandwidth, $\left.r_{0}=30 \mathrm{~mm}\right)$. (c) Reconstructed image using a nonpointlike detector (aperture size $2 a=3 \mathrm{~mm}, n=512, B=$ bandwidth, $r_{0}=30 \mathrm{~mm}$ ). (d) Comparison of the intensity profiles of the dots in the red square boxes in the radial direction, showing that the detector aperture size only slightly influences the radial resolution. (e) Comparison of the intensity profiles of the dots in the red square boxes in the tangential direction, showing that the detector aperture size has a significant impact on the tangential resolution.

\section{F. Detector focusing $\left(l_{n}\right)$}

The results presented so far are all based on unfocused detectors, which have a flat signal receiving surface, as shown in Fig. 14(a). In practice, focused detectors that exploit a curved surface or acoustic lens to detect ultrasound within a spatially confined region are also frequently employed in photoacoustic imaging. Examples of focused detectors include spherically focused detectors, which have maximum detection sensitivity at a point, and a cylindrically focused detector, which have maximum detection sensitivity at a line. Important parameters associated with focused detectors include the normalized focal length, $l_{n}$; the beam width at the focus, $w$; and the DOF. The normalized focal length, $l_{n}$, is defined as

$$
l_{n}=\frac{l_{f}}{z_{0}}
$$

where $l_{f}$ is the actual focal length and $z_{0}$ is the nearfield distance. For flat detectors, $l_{n}=1$ because $l_{f}=z_{0}$. For

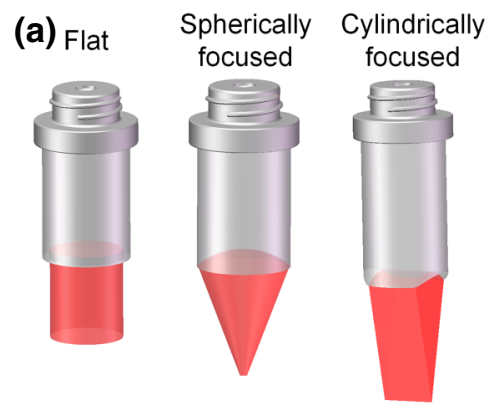

(b)

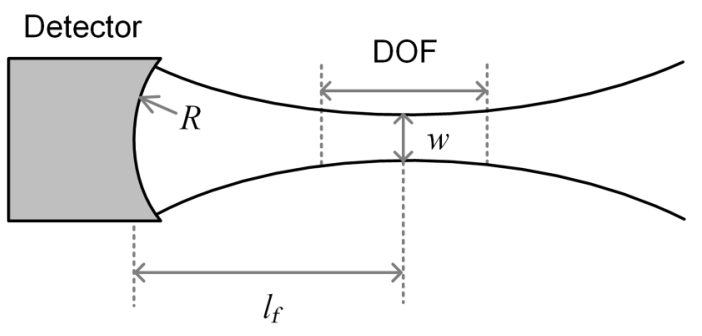

FIG. 14. Ultrasound detectors used in PAT. (a) Flat, spherically focused, and cylindrically focused detectors. (b) Schematic illustration of basic parameters associated with a focused detector. $R$, radius of curvature of the detector surface; $l_{f}$, focal length; $w$, beam width; DOF, depth of focus. 

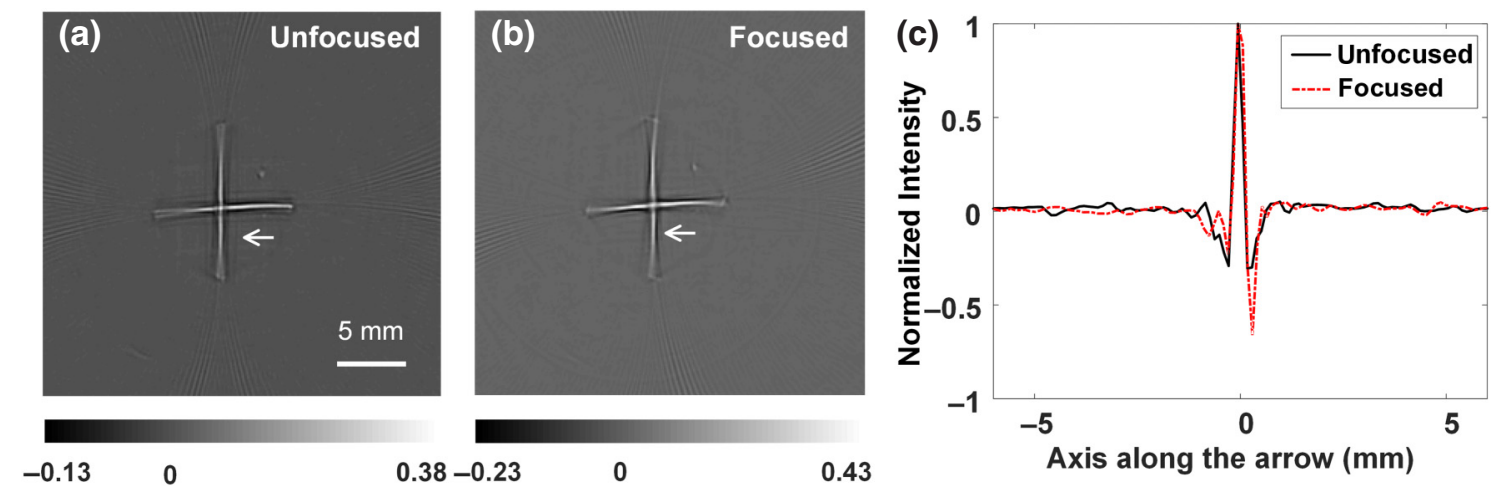

FIG. 15. Lateral resolution comparison of flat and focused detectors. (a) Hair cross image recorded using a $5 \mathrm{MHz}$ flat detector (Model: V326). (b) Same hair cross image recorded using a $5 \mathrm{MHz}$ focused detector (Model: V309, $l_{f}=50.8 \mathrm{~mm}$ ). (c) Intensity profile comparison of the rows indicated by the arrows in (a),(b). No significant lateral resolution differences are found.

focused detectors, $l_{n}$ is always smaller than one. The normalized focal length, $l_{n}$, is sometimes preferred, instead of the actual focal length, $l_{f}$. The $-6 \mathrm{~dB}$ beam width at the focus can be estimated as

$$
w_{-6 \mathrm{~dB}} \approx 1.03 \frac{l_{f}}{2 a} \lambda
$$

where $2 a$ is the aperture size of the detector and $\lambda$ is the ultrasound wavelength. In addition, the DOF of a focused detector can be written as

$$
\delta=z_{0} \frac{2 l_{n}^{2}}{1+0.5 l_{n}} .
$$

Taking the $5 \mathrm{MHz}$ spherically focused detector (Model: V309, $2 a=12.7 \mathrm{~mm}, l_{f}=50.8 \mathrm{~mm}$ ) as an example, the normalized focal length, $l_{n}$; beam width, $w$; and DOF are $0.38,1.2 \mathrm{~mm}$, and $32.6 \mathrm{~mm}$, respectively. Focused detectors are most frequently used in photoacoustic microscopy to detect sound waves within a spatially confined region for enhanced detection sensitivity.

One major consideration of employing focused detectors in PAT is to enhance elevational resolution in $3 \mathrm{D}$ imaging. After focusing, the ultrasound beam becomes much narrower compared with its aperture size and, therefore, could improve the elevational resolution significantly. Detector focusing will not significantly degrade the lateral resolution, which is determined by the center frequency, bandwidth, and aperture size of the detectors used. This is demonstrated in Fig. 15, where a human hair cross is imaged using an unfocused detector [Fig. 15(a)] and a focused detector [Fig. 15(b)]. The resultant images show comparable intensity profiles [Fig. 15(c)]; this means that there is no significant lateral resolution difference.

Focused detectors may improve imaging sensitivity at the focus region compared with flat detectors (Fig. 16) in PAT, but it depends on the ratio of the detector aperture size, $2 a$, and the detection radius, $r_{0}$. This is illustrated using a group of simulations based on a dot grid pattern (dot diameter, $250 \mu \mathrm{m}$ ), as shown in Fig. 17(a). When the detector aperture size, $2 a$, is relatively large compared with the detection radius, $r_{0}$, the sensitivity enhancement in the central region of the sample by using the focused detector is significant, as shown in Figs. 17(b) and 17(c); however, this is at the cost of sacrificing sensitivity in the nonfocused region. When the detector aperture size, $2 a$, is relatively small compared with the detection radius, $r_{0}$, the sensitivity enhancement by using the focused detector is not that significant, as shown in Figs. 17(d) and 17(e).
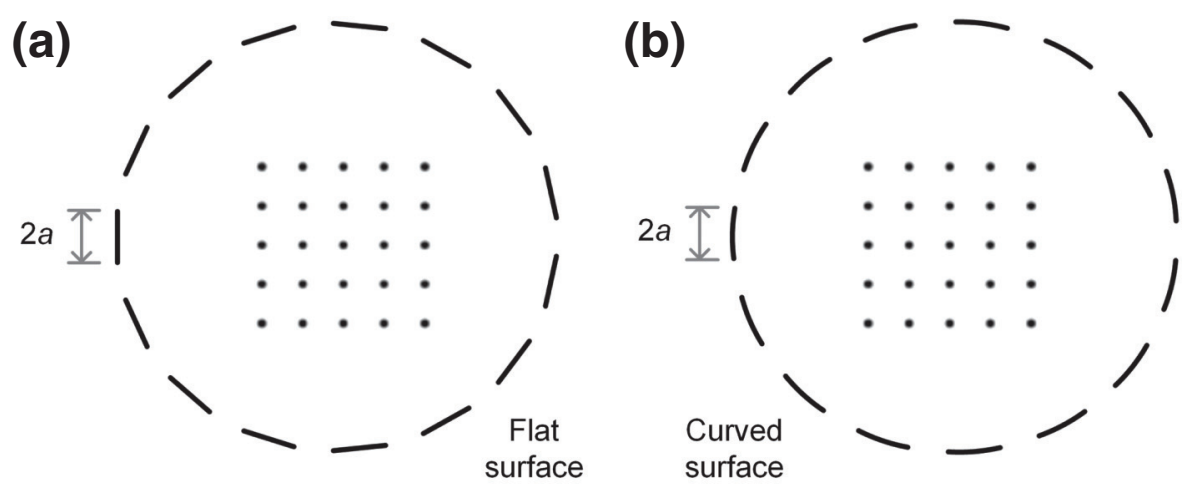

FIG. 16. Signal received using flat detectors (a) and focused detectors (b). 

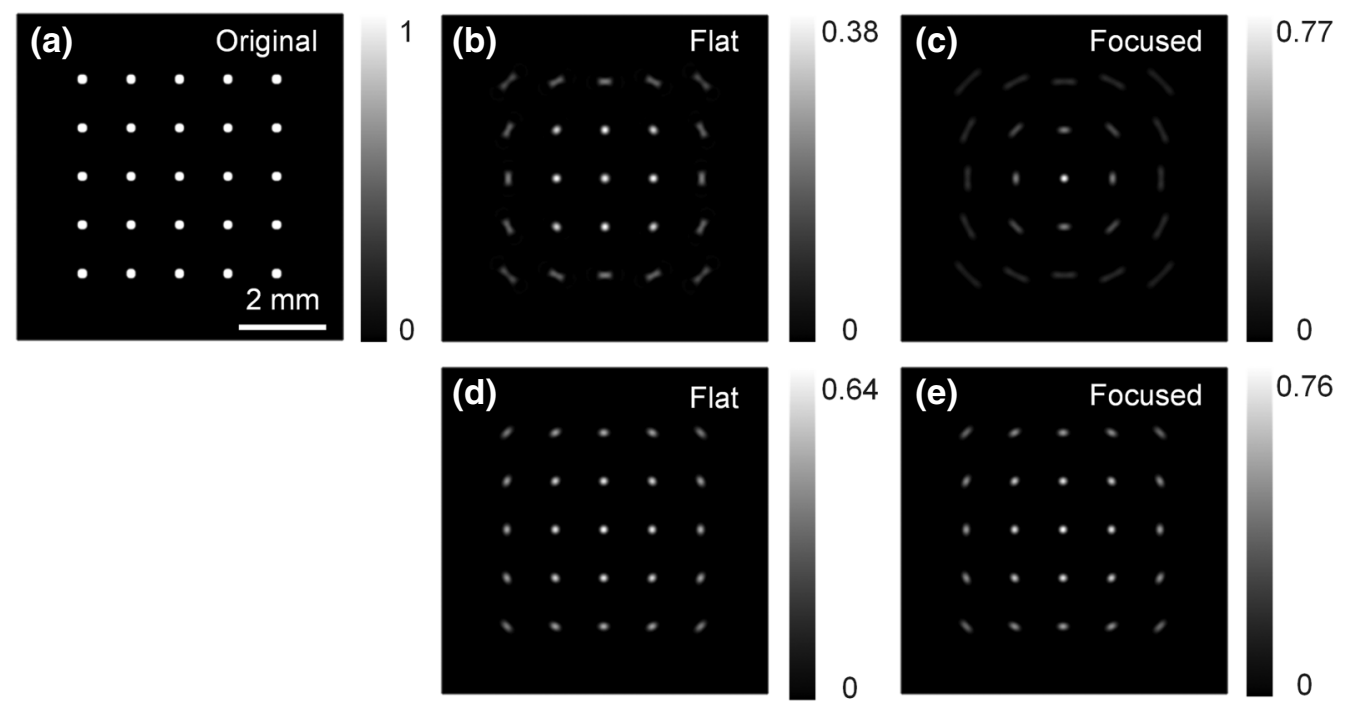

FIG. 17. The effect of detector focusing on image quality. (a) Dot grid phantom used in the simulation (dot diameter: $250 \mu$ m). (b),(c) imaging results using flat and focused detectors, respectively $\left(R=r_{0}=25 \mathrm{~mm}\right)$, when the aperture size of the detector, $2 a$, is relatively large compared with the detection radius $r_{0}$. Here, $2 a / r_{0}=0.24\left(2 a=6 \mathrm{~mm}, r_{0}=25 \mathrm{~mm}, f_{c}=5 \mathrm{MHz}, B=100 \%\right)$. (d),(e) imaging results using flat and focused detectors, respectively $\left(R=r_{0}=60 \mathrm{~mm}\right)$, when the aperture size of the detector, $2 a$, is relatively small compared with the detection radius, $r_{0}$. Here, $2 a / r_{0}=0.1\left(2 a=6 \mathrm{~mm}, r_{0}=60 \mathrm{~mm}, f_{c}=5 \mathrm{MHz}, B=100 \%\right)$. Notably, the reconstructed dots are slighted smaller than the original ones because their edges possess negative values after back-projection reconstruction, which are set to zero.

In this situation, flat and focused detectors produce very similar results. It is necessary to point out that the tangential resolution produced by the focused detector is worse than that produced by the flat detector, especially when $2 a / r_{0}$ is relatively large, as in the case shown in Figs. 17(b) and $17(\mathrm{c})$. This is because, for a fixed detector aperture size $2 a$, focused detectors have a larger surface area, which will degrade the tangential resolution more significantly. In this example, only simulations are performed because it is difficult to fabricate the dot grid phantom (dot diameter, $250 \mu \mathrm{m}$ ) using conventional printing methods.

\section{G. Detector alignment errors $(d \alpha$ and $d \theta)$}

Because a single element detector is used to rotate around the sample for signal acquisition in the prototype PAT scanner, correct alignment of the detector is essential for accurate image reconstruction. Two detector alignment errors, namely, the orientation error, $d \alpha$, and the scan step angle error, $d \theta$, are commonly present in real experiments. The detector orientation error means that the normal of the detector does not correctly point to the center of the detection surface and can be characterized by the angle $d \alpha$ between the surface normal and the radial directions, as shown in Fig. 18(a). The scan step angle error indicates that the actual position of the detector does not coincide with its ideal position during scanning and can be characterized by the angle $d \theta$ between the actual position and the ideal position, as shown in Fig. 18(b). The detector orientation error, $d \alpha$, can blur reconstructed images because of directional responses of nonpointlike detectors, as discussed in Sec. III F. The scan step angle error, $d \theta$, can also degrade the imaging quality because of inaccurate detector positions used for image reconstruction.
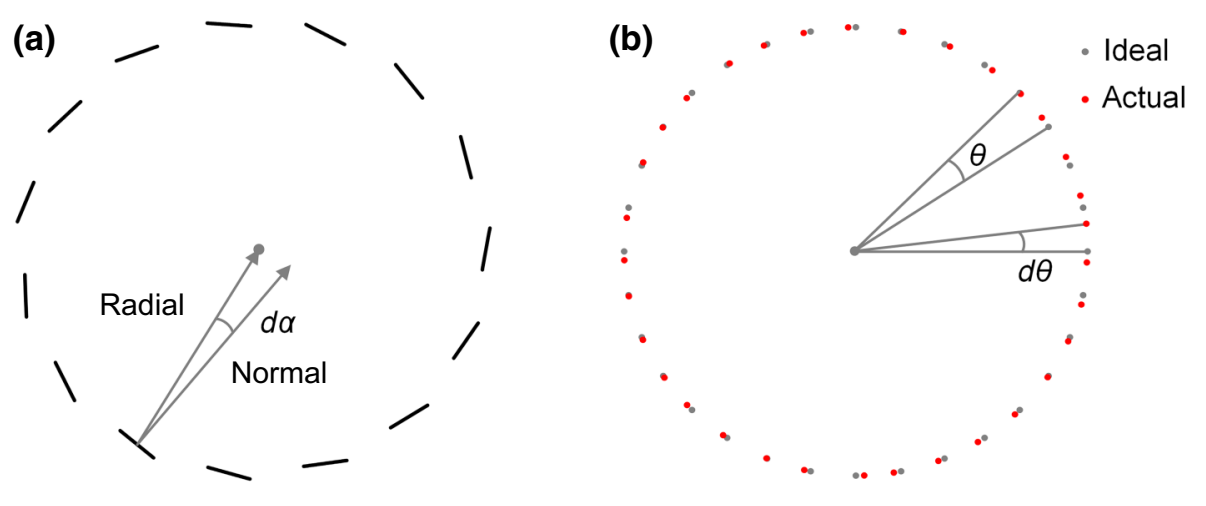

FIG. 18. Detector alignment errors. (a) Orientation error, $d \alpha$. (b) Step angle error, $d \theta$. Gray dots, ideal detector positions; red dots, actual detector positions. 

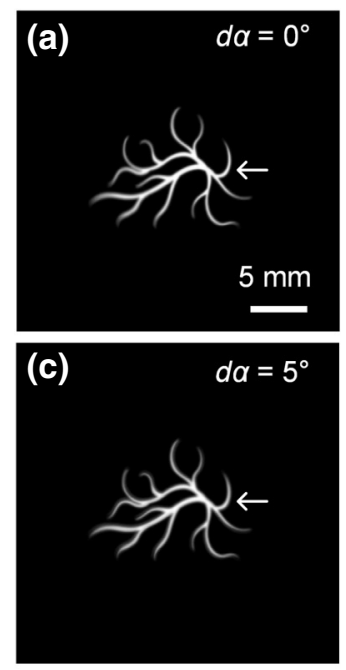
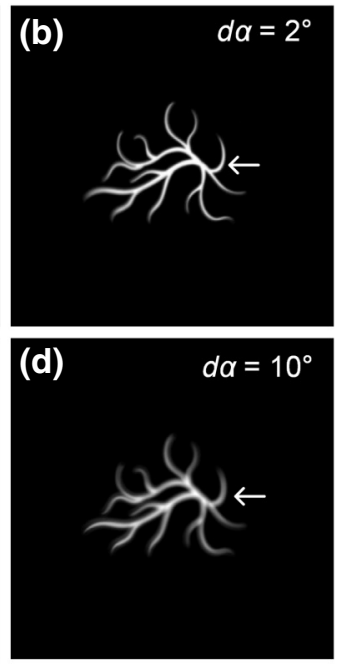

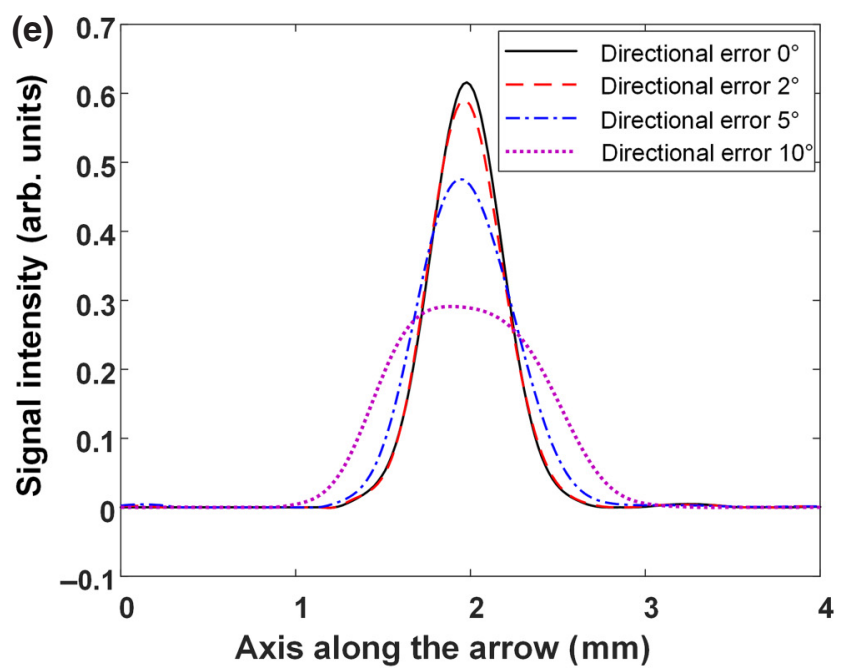

FIG. 19. The effect of detector orientation error on image quality. (a)-(d) Imaging results of the blood vessel phantom when the detector orientation errors $d \alpha$ are $0^{\circ}, 2^{\circ}, 5^{\circ}$, and $10^{\circ}$, respectively $\left(\Omega=360^{\circ}\right.$, flat detector, $2 a=6 \mathrm{~mm}, n=2048, B=$ full bandwidth, $r_{0}=120 \mathrm{~mm}$ ). (e) Intensity profiles of the vessel indicated by the arrows in (a)-(d).

Figure 19 is a group of simulations showing how the detector orientation error would affect the final image quality. Figures 19(a)-19(d) are imaging results of the blood vessels when values of $d \alpha$ are $0^{\circ}, 2^{\circ}, 5^{\circ}$, and $10^{\circ}$, respectively, and Fig. 19(e) is a comparison of intensity profiles of the vessel indicated by the arrows. It is seen that a larger orientation error, $d \alpha$, results in a more blurred reconstructed image. In practical situations, the detector should be correctly aligned to minimize possible blurring artifacts. In this example, only simulations are performed because it is difficult to precisely control the orientation error for small angles in the experiments.
Figure 20 is a set of simulations showing how the scan step angle error would affect the final image quality, when the total scan steps $n=256$ ( $1.4^{\circ}$ per scan step). Figures 20(a)-20(d) are imaging results of the blood vessel phantom, when $d \theta$ is uniformly distributed within the ranges of $\left[0^{\circ}, 0^{\circ}\right],\left[-1^{\circ}, 1^{\circ}\right],\left[-2^{\circ}, 2^{\circ}\right]$, and $\left[-3^{\circ}, 3^{\circ}\right]$, respectively. Figure 20(e) is the comparison of intensity profiles of the vessel indicated by the arrows. As expected, a larger scan step angle error, $d \theta$, results in more significant image reconstruction artifacts. In reality, the mechanical scanning system should be calibrated to avoid significant scan errors. In this example, only simulations are
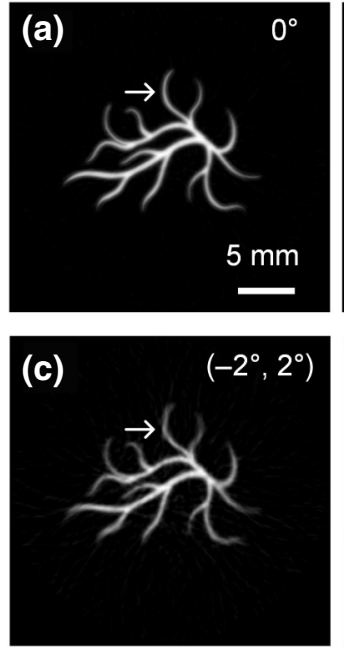
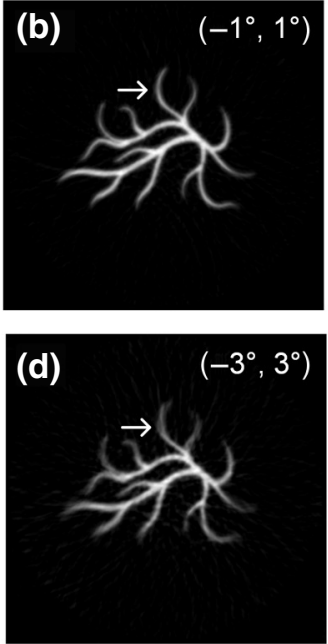

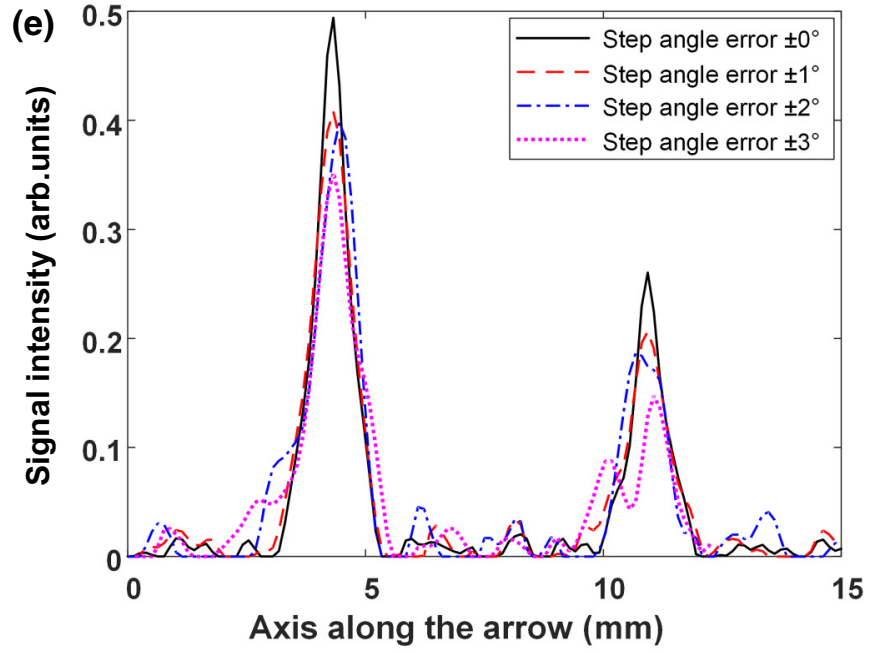

FIG. 20. The effect of step angle error on image quality. (a)-(d) Imaging results of the blood vessel phantom when the scan step angle errors, $d \theta$, are uniformly distributed within the ranges of $\left[0^{\circ}, 0^{\circ}\right],\left[-1^{\circ}, 1^{\circ}\right],\left[-2^{\circ}, 2^{\circ}\right]$, and $\left[-3^{\circ}, 3^{\circ}\right]$, respectively $\left(\Omega=360^{\circ}\right.$, $n=256$, point detector, $B=$ full bandwidth, $r_{0}=30 \mathrm{~mm}$ ). (e) Intensity profiles of the vessel indicated by the arrows in (a)-(d). 
performed because it is difficult to randomly control the scan step angle error in the experiments.

\section{CONCLUSION}

Here, we numerically and experimentally study eight system factors, which have significant impacts on the imaging performance of the prototype PAT scanner. The eight system factors can be grouped into three categories, namely, the detector arrangement group, the detector property group, and the detector alignment group.

In the detector arrangement group, factors including the detector view angle and the detector number should be considered. To achieve stable image reconstruction, the detection view angle needs not to be $2 \pi$ radian in $2 \mathrm{D}$ and $4 \pi$ steradian in $3 \mathrm{D}$. As long as the object is within the detection region, it can be stably recovered. For structures outside of the detection region, they can be recovered if the normal of the structure intersects with the detection surface. The detector number should be large enough to acquire sufficient projection data for image reconstruction in the inverse process, but the acquired projections may saturate if the detector number is too large. For 2D imaging, 256 detectors evenly distributed on a circle is expected to yield good image quality.

In the detector property group, factors including the detector center frequency, detector bandwidth, detector aperture size, and detector focusing effect should be considered. The detector center frequency should be chosen to match the frequency spectrum of the object being imaged to achieve maximum receiving sensitivity. Detectors with higher center frequencies produce sharper images. The bandwidth, together with the center frequency of a detector, determines the spatial resolving power of a PAT scanner. Broader bandwidth helps to recover both lowand high-frequency content of the object, while suppressing oscillation ringing artifacts. The aperture of a physical detector is not favored in practice because it degrades the accuracy of the mathematical model used for image reconstruction and endows the detector with a directional response. The final outcome is that the image resolution will be degraded, especially in the direction parallel with the detector aperture. In addition, compared with flat detectors, focused detectors can enhance signal detection sensitivity within focal regions and are commonly used in photoacoustic microscopy. When used in PAT, focused detectors could improve elevational resolution in 3D imaging, but have little impact on the lateral resolution.

In the detector alignment group, factors including the detector orientation error and the scan step angle error should be considered. Both errors can blur reconstructed images and produce artifacts, if large enough. Correct alignment of the detector is required to minimize reconstruction artifacts and produce high-quality images.
The conclusions obtained in this study are of general significance and can provide practical guidelines on the design of advanced PAT scanners with enhanced imaging performance.

\section{ACKNOWLEDGMENTS}

We would like to thank Tong Wang for preparing Fig. 11 and Chenxi Zhang for preparing Fig. 14. This work is supported by the National Natural Science Foundation of China (NSFC) under Grant No. 61705216, the Major Science and Technology Project of Anhui Province under Grant No. 18030801138, the Chinese Academy of Sciences (CAS) Pioneer Hundred Talents Program, and the startup fund of the University of Science and Technology of China (USTC).

[1] L. V. Wang and S. Hu, Photoacoustic tomography: In vivo imaging from organelles to organs, Science 335, 1458 (2012).

[2] C. Tian, Z. Xie, M. L. Fabiilli, and X. Wang, Imaging and sensing based on dual-pulse nonlinear photoacoustic contrast: A preliminary study on fatty liver, Opt. Lett. 40, 2253 (2015).

[3] C. Tian, Z. Xie, M. L. Fabiilli, S. Liu, C. Wang, Q. Cheng, and $\mathrm{X}$. Wang, Dual-pulse nonlinear photoacoustic technique: A practical investigation, Biomed. Opt. Express 6, 2923 (2015).

[4] T. Feng, K. M. Kozloff, C. Tian, J. E. Perosky, Y.-S. Hsiao, S. Du, J. Yuan, C. X. Deng, and X. Wang, Bone assessment via thermal photo-acoustic measurements, Opt. Lett. 40, 1721 (2015).

[5] H. Wang, S. Liu, T. Wang, C. Zhang, T. Feng, and C. Tian, Three-dimensional interventional photoacoustic imaging for biopsy needle guidance with a linear array transducer, J. Biophotonics, e2300 (2019).

[6] T. Köker, N. Tang, C. Tian, W. Zhang, X. Wang, R. Martel, and F. Pinaud, Cellular imaging by targeted assembly of hot-spot SERS and photoacoustic nanoprobes using splitfluorescent protein scaffolds, Nat. Commun. 9, 607 (2018).

[7] L. Wang, K. Maslov, and L. V. Wang, Single-cell label-free photoacoustic flowoxigraphy in vivo, Proc. Natl. Acad. Sci. U.S.A. 110, 5759 (2013).

[8] E. M. Strohm, M. J. Moore, and M. C. Kolios, Single cell photoacoustic microscopy: A review, IEEE J. Sel. Top. Quantum Electron 22, 137 (2016).

[9] C. Tian, W. Qian, X. Shao, Z. Xie, X. Cheng, S. Liu, Q. Cheng, B. Liu, and X. Wang, Plasmonic nanoparticles with quantitatively controlled bioconjugation for photoacoustic imaging of live cancer cells, Adv. Sci. 3, 1600237 (2016).

[10] W. Li, R. Chen, J. Lv, H. Wang, Y. Liu, Y. Peng, Z. Qian, G. Fu, and L. Nie, In vivo photoacoustic imaging of brain injury and rehabilitation by high-efficient nearinfrared dye labeled mesenchymal stem cells with enhanced brain barrier permeability, Adv. Sci. 5, 1700277 (2018).

[11] J. Yao, L. Wang, J.-M. Yang, K. I. Maslov, T. T. Wong, L. Li, C.-H. Huang, J. Zou, and L. V. Wang, High-speed 
label-free functional photoacoustic microscopy of mouse brain in action, Nat. Methods 12, 407 (2015).

[12] R. Cao, J. Li, B. Ning, N. Sun, T. Wang, Z. Zuo, and $\mathrm{S}$. Hu, Functional and oxygen-metabolic photoacoustic microscopy of the awake mouse brain, NeuroImage 150, 77 (2017).

[13] G. P. Luke and S. Y. Emelianov, Label-free detection of lymph node metastases with US-guided functional photoacoustic imaging, Radiology 277, 435 (2015).

[14] J. A. Guggenheim, T. J. Allen, A. Plumb, E. Z. Zhang, M. Rodriguez-Justo, S. Punwani, and P. C. Beard, Photoacoustic imaging of human lymph nodes with endogenous lipid and hemoglobin contrast, J. Biomed. Opt. 20, 050504 (2015).

[15] J. Jo, C. Tian, G. Xu, J. Sarazin, E. Schiopu, G. Gandikota, and X. Wang, Photoacoustic tomography for human musculoskeletal imaging and inflammatory arthritis detection, Photoacoustics 12, 82 (2018).

[16] C. Tian, W. Zhang, A. Mordovanakis, X. Wang, and Y. M. Paulus, Noninvasive chorioretinal imaging in living rabbits using integrated photoacoustic microscopy and optical coherence tomography, Opt. Express 25, 15947 (2017).

[17] V. P. Nguyen, Y. Li, W. Qian, B. Liu, C. Tian, W. Zhang, Z. Huang, A. Ponduri, M. Tarnowski, X. Wang, and Y. M. Paulus, Contrast agent enhanced multimodal photoacoustic microscopy and optical coherence tomography for imaging of rabbit choroidal and retinal vessels in vivo, Sci. Rep. 9, 5945 (2019).

[18] American National Standards Institute, American National Standard for Safe Use of Lasers ANSI Z136.1-2007 (Laser Institute of America, Orlando, FL, USA, 2007).

[19] B. E. Treeby, E. Z. Zhang, and B. T. Cox, Photoacoustic tomography in absorbing acoustic media using time reversal, Inverse Probl. 26, 115003 (2010).

[20] M. Xu and L. V. Wang, Analytic explanation of spatial resolution related to bandwidth and detector aperture size in thermoacoustic or photoacoustic reconstruction, Phys. Rev. E 67, 056605 (2003).

[21] O. Scherzer, Handbook of Mathematical Methods in Imaging, Chapter 18 (Springer Science \& Business Media, New York, NY, USA, 2010).

[22] Y. Xu, L. V. Wang, G. Ambartsoumian, and P. Kuchment, Reconstructions in limited-view thermoacoustic tomography, Med. Phys. 31, 724 (2004).
[23] M. Haltmeier and G. Zangerl, Spatial resolution in photoacoustic tomography: Effects of detector size and detector bandwidth, Inverse Probl. 26, 125002 (2010).

[24] A. Dima, N. C. Burton, and V. Ntziachristos, Multispectral optoacoustic tomography at 64,128 , and 256 channels, J. Biomed. Opt. 19, 036021 (2014).

[25] R. A. Kruger, P. Liu, and C. R. Appledorn, Photoacoustic ultrasound (PAUS)-reconstruction tomography, Med. Phys. 22, 1605 (1995).

[26] X. Wang, Y. Pang, G. Ku, X. Xie, G. Stoica, and L. V. Wang, Noninvasive laser-induced photoacoustic tomography for structural and functional in vivo imaging of the brain, Nat. Biotechnol. 21, 803 (2003).

[27] A. Sharma, S. K. Kalva, and M. Pramanik, A comparative study of continuous versus stop-and-go scanning in circular scanning photoacoustic tomography, IEEE J. Sel. Top. Quantum Electron 25, 1 (2019).

[28] S. E. Bohndiek, S. Bodapati, D. Van De Sompel, S.-R. Kothapalli, and S. S. Gambhir, Development and application of stable phantoms for the evaluation of photoacoustic imaging instruments, PLoS One 8, e75533 (2013).

[29] B. E. Treeby and B. T, Cox, k-Wave: MATLAB toolbox for the simulation and reconstruction of photoacoustic wave fields, J. Biomed. Opt. 15, 021314 (2010).

[30] M. Xu and L. V. Wang, Universal back-projection algorithm for photoacoustic computed tomography, Phys. Rev. E 71, 016706 (2005).

[31] V. G. Andreev, A. A. Karabutov, and A. A. Oraevsky, Detection of ultrawide-band ultrasound pulses in optoacoustic tomography, IEEE Trans. Ultrason. Ferroelectr. Freq. Control 50, 1383 (2003).

[32] G. Ku, X. Wang, G. Stoica, and L. V. Wang, Multiplebandwidth photoacoustic tomography, Phys. Med. Biol. 49, 1329 (2004).

[33] Z. Xie, S.-L. Chen, T. Ling, L. J. Guo, P. L. Carson, and X. Wang, Pure optical photoacoustic microscopy, Opt. Express 19, 9027 (2011).

[34] S.-L. Chen, S.-W. Huang, T. Ling, S. Ashkenazi, and L. J. Guo, Polymer microring resonators for high-sensitivity and wideband photoacoustic imaging, IEEE Trans. Ultrason. Ferroelectr. Freq. Control 56, 2482 (2009).

[35] X. Yang, M.-L. Li, and L. V. Wang, Ring-based ultrasonic virtual point detector with applications to photoacoustic tomography, Appl. Phys. Lett. 90, 251103 (2007). 